\title{
Schleimhautplastik mit autologer oraler Mukosa zur Therapie des Symblepharon bei Morbus Wegener - eine Kasuistik ${ }^{1}$
}

Birgit Orth', Uwe Peter Press², Horst Hübner², Uwe Pleyer ${ }^{1}$

${ }^{1}$ Augenklinik Charité, Campus Virchow-Klinikum der Humboldt Universität zu Berlin, 13344 Berlin (Dir.: Prof. Dr. Dr. C. Hartmann)

${ }^{2}$ Augenklinik, Krankenhaus der Barmherzigen Brüder, Trier (Leitung: Prof. Dr. M. Wenzel)

In dem Artikel „Schleimhautplastik mit autologer oraler Mukosa zur Therapie des Symblepharon bei Morbus Wegener eine Kasuistik“, Klin Monatsbl Augenheilkd 2001; 218: 514517, wurde in der Klinikzeile versehentlich Prof. Wenzel als alleiniger Chefarzt der Abteilung genannt.

Richtig ist:

Chefärzte im Kollegialsystem im Krankenhaus der Barmherzigen Brüder Trier sind

Prof. Dr. M. Wenzel, Abteilung für Allgemeine Ophthalmologie und Dr. U. P. Press, Abteilung für Plastisch Rekonstruktive Lid-, Orbita- und Tränenwegschirurgie, Strabologie.

Wir bitten um Entschuldigung. 